$03,09,12$

\title{
Спектры отражения и фотоотражения структур с квантовыми ямами на основе ZnO
}

\author{
(C) А.М. Клюев ${ }^{1}$, Н.Г. Философов ${ }^{2}$, А.Ю. Серов ${ }^{2}$, В.Ф. Агекян ${ }^{2}$, С. Morhain $^{3}$, В.П. Кочерешко ${ }^{1, \oplus}$ \\ ${ }^{1}$ Физико-технический институт им. А.Ф. Иофрфе РАН, \\ Санкт-Петербург, Россия \\ ${ }^{2}$ Санкт-Петербургский государственный университет \\ Санкт-Петербург, Россия \\ ${ }^{3}$ Centre de Recherche sur l'Hétéro-Epitaxie et ses Applications CRHEA-CNRS \\ ฯ E-mail: Vladimir.Kochereshko@mail.ioffe.ru
}

Поступила в Редакцию 13 июня 2020 г.

В окончательной редакции 13 июня 2020 г.

Принята к публикации 16 июня 2020 г.

Исследованы спектры отражения и модуляционного фотоотражения от эпитаксиальных слоев $\mathrm{ZnO}$ и $\mathrm{Zn}_{1-x} \mathrm{Mg}_{x} \mathrm{O}$, а также квантовых ям $\mathrm{ZnO} / \mathrm{Zn}_{1-x} \mathrm{Mg}_{x} \mathrm{O}$ при нормальном и наклонном падении света. Сравнение экспериментально измеренных и расчетных спектров позволило уточнить некоторые параметры экситонов в этих структурах и уточнить порядок следования энергетических зон в ZnO.

Ключевые слова: гетероструктуры, квантовые ямы, экситоны, спектроскопия.

DOI: 10.21883/FTT.2020.11.50052.127

\section{1. Введение}

Широкозонные полупроводниковые соединения, такие как $\mathrm{ZnO}, \mathrm{GaN}, \mathrm{MgO}$, AlN представляются чрезвычайно перспективными материалами для оптоэлектроники. Благодаря большой ширине запрещенной зоны такие структуры удобны для изготовления полупроводниковых лазеров, работающих в ультрафиолетовом диапазоне и сверхвысоковольтных транзисторов. Очень часто в таких структурах благодаря пьезоэффекту появляется встроенное электрическое поле напряженностью до нескольких миллионов вольт на сантиметр [1]. В то же время в этих материалах наблюдается и достаточно большая концентрация примесей, достигающая $10^{18} \mathrm{~cm}^{-3}$, что приводит к частичной экранировке этого встроенного поля. В результате величина встроенного поля меняется в зависимости от вида структуры и даже от условий, при которых они исследуются. Как следствие, многие свойства экситонных и трионных состояний, зависят от встроенного электрического поля и оказываются плохо контролируемыми. Дело доходит до того, что даже интерпретация экситонных и трионных линий в спектрах таких структур оказывается неоднозначной. Имеются значительные расхождения и в опубликованных величинах параметров экситона [2]. Более того, с шестидесятых годов $[3,4]$ и до сих пор ведется дискуссия о порядке следования энергетических зон в оксиде цинка и гетероструктурах на основе $\mathrm{ZnO}$ [5]. Это обосновывает актуальность исследований этих материалов.

В работе экспериментально и теоретически исследовались спектры отражения и фотоотражения при нормальном и наклонном падении света от гетероструктур, содержащих как слои $\mathrm{ZnO}$ и $\mathrm{Zn}_{1-x} \mathrm{Mg}_{x} \mathrm{O}$, так и квантовые ямы $\mathrm{ZnO} / \mathrm{Zn}_{1-x} \mathrm{Mg}_{x} \mathrm{O}$ с содержанием $\mathrm{Mg} x=0.22$.

\section{2. Эксперимент и результаты}

На подложке сапфира был выращен буферный слой $\mathrm{ZnO}$ толщиной порядка $1 \mathrm{mkm}$, затем выращивался барьерный слой $\mathrm{Zn}_{1-x} \mathrm{Mg}_{x} \mathrm{O}$ толщиной $200 \mathrm{~nm}$, следом за ним выращивались квантовая яма $\mathrm{ZnO}$ толщиной $7.1 \mathrm{~nm}$, еще один барьерный слой $\mathrm{Zn}_{1-x} \mathrm{Mg}_{x} \mathrm{O}$ толщиной $200 \mathrm{~nm}$, квантовая яма $\mathrm{ZnO}$ толщиной $2.7 \mathrm{~nm}$ и покрывающий слой $\mathrm{Zn}_{1-x} \mathrm{Mg}_{x} \mathrm{O}$ толщиной $200 \mathrm{~nm}$.

Спектры отражения регистрировались с помощью галогенной лампы и монохроматора МДР-406, оснащенного фотоумножителем, в режиме счета фотонов, температура образца во всех экспериментах составляла $5 \mathrm{~K}$. Спектры фотоотражения регистрировались при дополнительной подсветке образца импульсами лазера с длиной волны $332 \mathrm{~nm} \mathrm{c} \mathrm{последующим} \mathrm{синхронным}$ детектированием сигнала.

На рис. 1, $a$ представлен спектр отражения (сплошная кривая), снятый при нормальном падении света на образец при температуре $5 \mathrm{~K}$. В спектре отчетливо наблюдаются экситонные линии: в слое $\mathrm{ZnO}$, на энергии $3.370 \mathrm{eV}$, в слое $\mathrm{Zn}_{1-x} \mathrm{Mg}_{x} \mathrm{O}$, на энергии $3.65 \mathrm{eV}$, и квантовой яме (КЯ) шириной $2.7 \mathrm{~nm}$ на энергии $3.444 \mathrm{eV}$. Эти линии были идентифицированы в работе [6] путем сравнения спектров отражения и фотолюминесценции. Однако, идентификация экситонных линий от КЯ, шириной $7.1 \mathrm{~nm}$ вызвала затруднения. Так в работе [7] считали, что линия экситона в широкой КЯ сильно сдвинута пьезоэлектрическим полем в длинноволновую часть спектра в область $3.2 \mathrm{eV}$, а в работе [8] предполагалось, что сигнал фотолюминесценции в этой области спектра связан с рекомбинацией донорно-акцепторных пар. 

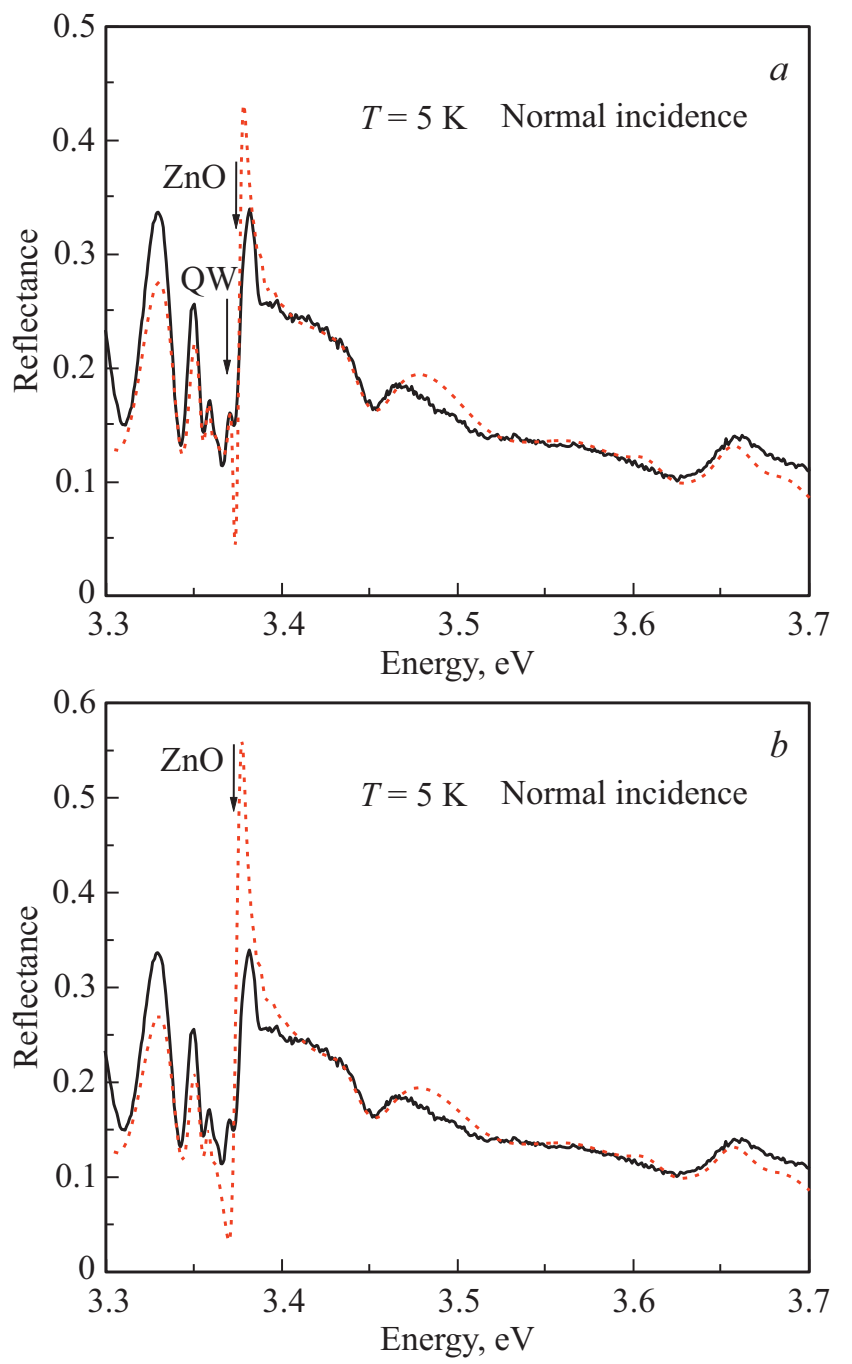

Pис. 1. a) Экспериментально измеренный спектр отражения (сплошная кривая) при нормальном падении света, $T=5 \mathrm{~K}$. Стрелками показаны энергии экситонных резонансов в слое оксида цинка $(\mathrm{ZnO})$ и квантовой яме шириной $7.1 \mathrm{~nm}(\mathrm{QW})$. Расчет спектра отражения (пунктирная кривая) с параметрами, приведенными в таблице. b) Экспериментально измеренный спектр отражения (сплошная кривая) при нормальном падении света, $T=5 \mathrm{~K}$. Расчет спектра отражения (пунктирная кривая) без учета квантовой ямы.

Помимо этих экситонных линий в длинноволновой части спектра отражения наблюдается интерференционная картина, связанная с квантованием поляритонов в слое $\mathrm{ZnO}$. Еще одно обстоятельство обращает на себя внимание: наблюдаемая в коротковолновой части спектра линия экситонного отражения имеет „перевернутую“ форму. Подобное вращение контура экситонного отражения происходит при интерференции света в толстом безэкситонном слое $[9,10]$ вблизи поверхности.

На этом же рисунке представлен расчет спектра отражения от исследуемой структуры с учетом вклада обеих КЯ. Видно, достаточно хорошее совпадение расчетного спектра с экспериментально измеренным.
Для того чтобы выявить вклад КЯ шириной $7.1 \mathrm{~nm}$ в спектр отражения, мы провели расчет этого спектра с учетом и без учета этой КЯ, при этом все остальные параметры расчета не менялись. Видно, что в области энергий $3.36 \mathrm{eV}$ не удается добиться удовлетворительного совпадения расчетного спектра с измеренным без учета вклада от этой КЯ (рис. $1, b)$.

Таким образом, вопреки утверждению многих статей, длинноволновый сдвиг экситонных линий в КЯ в пьезоэлектрическом поле данной структуры не так уж велик и составляет всего несколько $\mathrm{meV}$. Малая величина штарковского сдвига экситонных линий, вероятно, обусловлена экранированием пьезоэлектрического поля свободными носителями, пришедшими с доноров, а также рожденными светом в процессе эксперимента.

Для дополнительной проверки этого факта были измерены спектры модулированного фотоотражения $d R / R$ (рис. 2). В этом эксперименте для надбарьерной подсветки использовался лазер с энергией кванта $3.815 \mathrm{eV}$ и строилась разность сигналов с подсветкой и без нее

$$
\frac{d R}{R}=\frac{R\left(\omega_{0}+\delta\right)-R\left(\omega_{0}-\delta\right)}{R\left(\omega_{0}+\delta\right)+R\left(\omega_{0}-\delta\right)} .
$$

Здесь: $R$ - коэффициент отражения, $\omega_{0}$ - частота экситонного резонанса, $\delta$ - вариация резонансной частоты. В расчете мы считали, что $\delta=1 \mathrm{meV}$.

На рис. 2 видны яркие особенности в области энергий $3.376 \mathrm{eV}$ и интерференционная картина в области $3.340 \mathrm{eV}$. На этом же рисунке представлен расчетный спектр фотоотражения в предположении, что главный вклад в спектр происходит от изменения резонансной частоты экситона в толстом слое $\mathrm{ZnO}$ и в КЯ шириной $7.1 \mathrm{~nm}$. Расчет показал, что при изменении других экситонных параметров, таких как сила осциллятора и/или

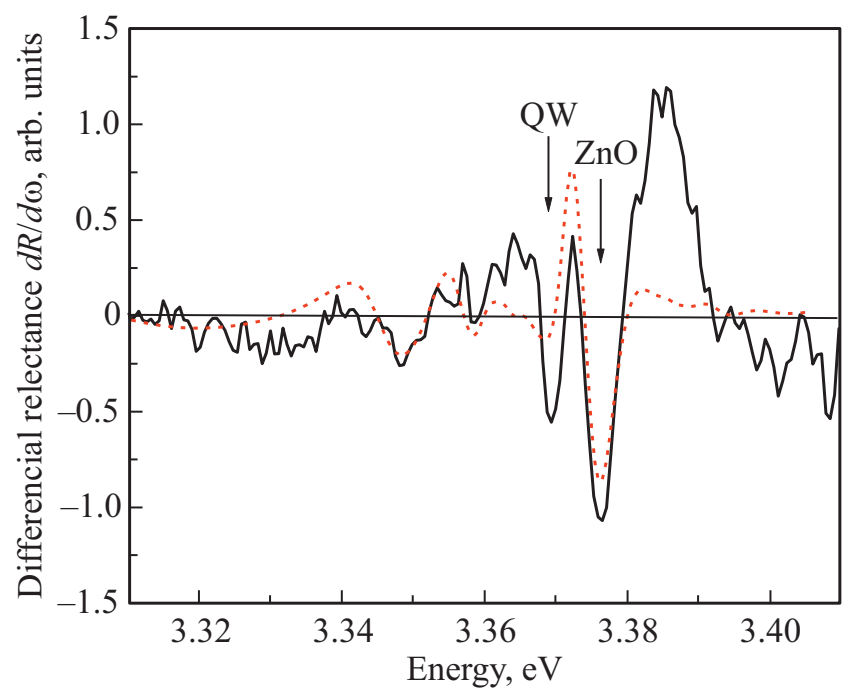

Рис. 2. Спектральная зависимость сигнала дифференциального фотоотражения при дополнительной надбарьерной подсветке. Сплошная кривя - эксперимент, пунктирная кривая расчет. 


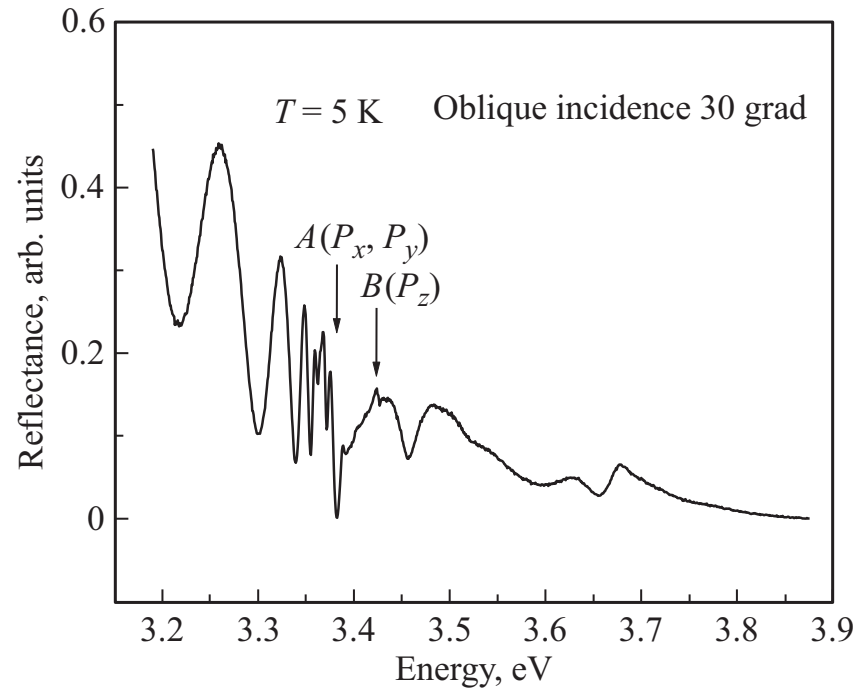

Pис. 3. Спектр отражения, зарегистрированный при наклонном падении света, $T=5 \mathrm{~K}$. Стрелками указаны экситонные резонансы, поляризованные перпендикулярно гексагональной оси $\left(P_{x}, P_{y}\right)$ и вдоль гексагональной оси $\left(P_{z}\right)$.

затухание не удается получить достаточно хорошее совпадение расчета с экспериментом.

Для дальнейшего уточнения параметров были измерены спектры отражения при наклонном падении света под углом $\sim 30$ градусов. Неожиданно оказалось, что при наклонном падении в спектре появляется еще одна линия отражения с энергией $3.424 \mathrm{eV}$ (рис. 3). При этом общий вид спектра соответствует случаю нормального падения. Очевидно, что эта новая линия связана с экситонным переходом, поляризованным вдоль гексагональной оси. Это указывает на то, что в данной структуре реализуется нормальный порядок зон $\left(E_{g}^{A}<E_{g}^{B}<E_{g}^{C}\right)$, предложенный в работе [3]. Возможно, что различие в расположении зон, представленное в разных публикациях, связано с различием механических напряжений в структурах, выращенных в разных условиях (на подложке и без подложки).

\section{3. Теория}

В расчете рассматривалась структура, состоящая из следующих слоев: подложка из сапфира, слой $\mathrm{ZnO}$ толщиной порядка $1 \mathrm{mkm}$, затем слой $\mathrm{Zn}_{1-x} \mathrm{Mg}_{x} \mathrm{O}$ толщиной $200 \mathrm{~nm}$, за ним КЯ толщиной $7.1 \mathrm{~nm}$, еще один барьерный слой толщиной $200 \mathrm{~nm}$, КЯ толщиной $2.7 \mathrm{~nm}$ и покрывающий слой толщиной $200 \mathrm{~nm}$.

Считалось, что показатель преломления света в сапфире равен $n_{S a}=1.7$, фоновые диэлектрические проницаемости в $\mathrm{ZnO}$ и $\mathrm{Zn}_{1-x} \mathrm{Mg}_{x} \mathrm{O}$ одинаковы и равны $\varepsilon_{0}=4.2[11]$.

Распространение света в слоях рассчитывалось методом матриц переноса. Мы считали, что в барьерных слоях $\mathrm{Zn}_{1-x} \mathrm{Mg}_{x} \mathrm{O}$ экситонное затухание велико
Параметры экситонов $\left(\hbar \omega_{0}-\right.$ резонансная частота экситона, $\hbar \omega_{L T}-$ продольно поперечное расщепление, $\hbar \Gamma-$ затухание, $\varepsilon_{0}-$ фоновая диэлектрическая проницаемость)

\begin{tabular}{c|c|c|c|c}
\hline Параметр & $\hbar \omega_{0}, \mathrm{eV}$ & $\hbar \omega_{L T}^{*}, \mathrm{eV}$ & $\hbar \Gamma, \mathrm{meV}$ & $\varepsilon_{0}$ \\
\hline $\mathrm{ZnO}$ & 3.373 & 6.2 & 1.9 & 3.82 \\
$\mathrm{Zn}_{1-x} \mathrm{Mg}_{x} \mathrm{O}$ & 3.650 & 9.5 & 15 & 3.75 \\
$\mathrm{QW}$ & 3.370 & 14.0 & 2.1 & 3.82 \\
$7.1 \mathrm{~nm}$ & & &
\end{tabular}

Пр и м е ча н и е. Для квантовой ямы приведена величина эффективного продольно-поперечного расщепления, полученная при переходе от нелокального отклика к локальному [12].

и пространственную дисперсию в них учитывать не нужно. Это предположение подтверждается большим неоднородным уширением экситонной линии отражения в барьерах. Отражение света от КЯ рассматривалось в модели нелокального отклика [12]. Пространственная дисперсия учитывалась только для слоя $\mathrm{ZnO}$. В таблице представлены параметры экситонов, которые использовались в расчете.

При теоретическом описании „перевернутого“ контура отражения (рис. 4) в барьерном слое мы считали, что происхождение безэкситонного слоя связано с наличием вблизи поверхности электрического поля, вызванного ионизованными примесями в приповерхностной области кристалла. В этом поле затухание экситона сильно возрастает, что и приводит к перевороту контура экситонного отражения.

Предположим, что концентрация ионизованных примесей вблизи поверхности равна $\left(N_{D}-N_{A}\right)$, плотность заряда этих примесей $\rho=e\left(N_{D}-N_{A}\right)$. Тогда напряжен-

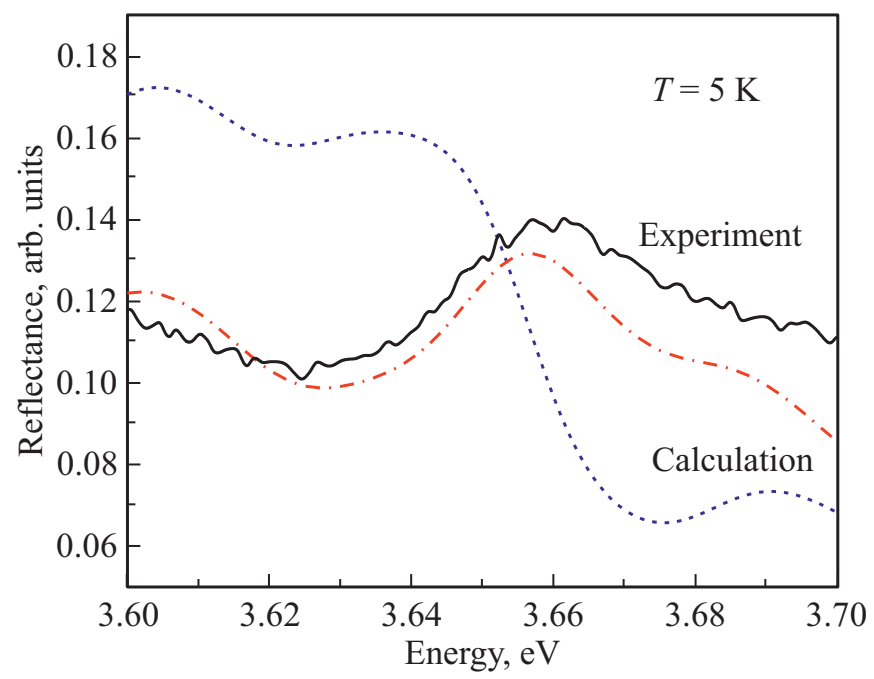

Рис. 4. Измеренный спектр отражения в области экситона барьерного слоя $\mathrm{Zn}_{1-x} \mathrm{Mg}_{x} \mathrm{O}$ (сплошная кривая) и рассчитанные спектры отражения без учета приповерхностного электрического поля (пунктирная кривая) и с учетом приверхностного поля (штрих-пунктирная кривая). 
ность поля, создаваемого этими примесями, будет

$$
E(z)=\frac{4 \pi e}{\varepsilon_{0}}\left(N_{D}-N_{A}\right)\left(z-z_{0}\right), \quad E(0)=E_{s} .
$$

Здесь: $z_{0}$ - длина экранирования поверхностных состояний свободными носителями, $E_{s}-$ величина поля на поверхности.

То есть мы считали, что в приповерхностной области поле меняется линейно, и экситонное затухание также линейно зависит от поля.

Для расчета экситонного отражения с учетом приверхностного поля использовалась многослойная аппроксимация безэкситонного слоя. Приповерхностная область разбивалась на слои толщиной $1 \mathrm{~nm}$ и в каждом слое задавались параметры экситона. Мы считали, что сила осциллятора и резонансная частота не меняются от слоя к слою, а затухание линейно растет при приближении к поверхности. Такая зависимость подтверждается расчетами влияния электрического поля на атом водорода и экситон $[10,13]$. Сравнение экспериментально измеренного и расчетного контура отражения экситона в слое $\mathrm{Zn}_{1-x} \mathrm{Mg}_{x} \mathrm{O}$ представлено на рис. 4 с учетом и без учета приповерхностного электрического поля.

\section{4. Обсуждение и выводы}

В настоящей работе в расчете спектров экситонного отражения мы не учитывали пространственную дисперсию в слоях $\mathrm{Zn}_{1-x} \mathrm{Mg}_{x} \mathrm{O}$. Это связано с тем, что, во-первых, в этих слоях мы не наблюдаем размерного квантования поляритонов, во-вторых, линия отражения экситона имеет большое неоднородное уширение, что указывает на сильные флуктуации ширины запрещенной зоны в твердом растворе $\mathrm{Zn}_{1-x} \mathrm{Mg}_{x} \mathrm{O}$. Следовательно, затухание экситона в этих слоях велико и превосходит величину продольно-поперечного расщепления. В этом случае пространственную дисперсию учитывать не нужно [12]. Действительно, рассогласование решеток $\mathrm{ZnO}$ и $\mathrm{MgO}$ достигает $\sim 10 \%$, и такой твердый раствор должен быть сильно неоднородным.

Качество слоев $\mathrm{ZnO}$ гораздо выше, на это указывает интерференционная структура с узкими линиями. В этом случае учет пространственной дисперсии необходим. Экситоны в квантовых ямах мы рассматривали в модели нелокального отклика.

Из сравнения экспериментально измеренного и расчетного спектров было установлено, что экситонный резонанс в КЯ $7.1 \mathrm{~nm}$ находится на энергии $3.37 \mathrm{eV}$. Это указывает на эффективное экранирование пьезоэлектрического поля свободными носителями. Действительно, концентрация примесей в нашей структуре составляет $\left(N_{D}-N_{A}\right) \approx 3 \cdot 10^{17} \mathrm{~cm}^{-3}$, такой концентрации достаточно для экранирования встроенного поля уже на расстояниях $\sim 30 \mathrm{~nm}[6]$. Были установлены величины сил осциллятора (продольно- поперечного расщепления) и затухания экситона в слоях $\mathrm{ZnO}, \mathrm{Zn}_{1-x} \mathrm{Mg}_{x} \mathrm{O}$ и в КЯ.
Спектр отражения, снятый при наклонном падении, указывает на то, что в нашей структуре реализуется нормальный порядок валентных зон в слое $\mathrm{ZnO}$.

\section{Финансирование работы}

ФНГ, АЮС и ВФА благодарят проект СПбГУ INI_2019, ID: 37688845 за частичную поддержку работы.

\section{Конфликт интересов}

Авторы заявляют, что у них нет конфликта интересов.

\section{Список литературы}

[1] Tomoki Abe, Tatsuya Motoyama, Masaya Yamamoto, Atsushi Yamamoto, Shohei Iwagashita, Hirofumi Kasada, Koshi Ando, and Kunio Ichino. Phys. Status Solidi C 13, 7-9, 602 (2016).

[2] Ü. Özgür, Ya.I. Alivov, C. Liu, A. Teke, M.A. Reshchikov, S. Doğan, V. Avrutin, S.-J. Cho, H. Morkoç. J. Appl. Phys. 98, 041301 (2005).

[3] D.G. Thomas. J. Phys. Chem. Solids 15, 86 (1960).

[4] Y.S. Park, C.W. Litton, T.C. Collins, D.C. Reynolds. Phys. Rev. 143, 512 (1966).

[5] J. Puls, S. Sadofev, F. Henneberger. Phys. Rev. B 85, 041307(R) (2012).

[6] M.N. Bataev, N.G. Filosofov, A.Yu. Serov, V.F. Agekyan, C. Mohrain, V.P. Kochereshko. Phys. Solid State, 60, 2491 (2018).

[7] C. Morhain, T. Bretagnon, P. Lefebvre, X. Tang, P. Valvin, T. Guillet, B. Gil, T. Taliercio, M. Teisseire-Doninelli, B. Vinter, C. Deparis. Phys. Rev. B 72, 241305R (2005).

[8] Thierry Bretagnon. Turkish J. Phys. 38, 420 (2014).

[9] Е.Л. Ивченко, П.С. Копьев, В.П. Кочерешко, И.Н. Уральцев, Д.Р. Яковлев, С.В. Иванов, Б.Я. Мельцер, М.А. Калитиевский. ФТП 22, 784 (1988).

[10] В.А. Киселев, Б.В. Новиков, А.Е. Чередниченко. Экситонная спектроскопия приповерхностной области полупроводников. Изд-во СПб ун-та, СПб (2003).

[11] Landolt-Bornstein „Numerical Data and Functional Relationship in Science and Technology“ / Ed. W. Martienssen. Group III: Condensed Matter, Springer (2000).

[12] E.L. Ivchenko. Optical Spectroscopy of Semiconductor Nanostructures. Alpha Science Int., Harrow, UK (2005).

[13] V.V. Kolosov. J. Phys. B 20, 2359 (1987).

Редактор К.В. Емцев 